\title{
The influence of Coulomb on the liquid-gas phase transition and nuclear multifragmentation
}

\author{
F.Gulminelli( ${ }^{(1)}, \mathrm{Ph}_{\text {CChomaz }}^{(2)}$, Al. H. Raduta ${ }^{(3)}$, Ad. R. Raduta ${ }^{(3)}$ \\ (1) LPC Caen (IN2P3-CNRS/Ensicaen et Université),F-14050 Caen Cédex,France \\ (2) GANIL (DSM-CEA/IN2P3-CNRS),B.P.5027 F-14021 Caen Cédex,France \\ (3) National Institute of Physics and Nuclear Engineering, Bucharest, POB MG6, Romania
}

\begin{abstract}
The liquid-gas phase transition is analyzed from the topologic properties of the event distribution in the obervables space. A multi-canonical formalism allows to directly relate the standard phase transition with neutral particles to the case where the non saturating Coulomb interaction is present, and to interpret the effect of Coulomb as a deformation of the probability distributions and a rotation of the order parameter. This formalism is applied to a statistical multifragmentation model and consequences for the nuclear multifragmentation phase transitions are drawn.
\end{abstract}

PACS numbers: 24.60.-k,21.10.Sf,68.18.Jk,03.65.Vf

Since the first pioneering multifragmentation experiments [1], the break-up of a hot nucleus above about $3 \mathrm{~A} . \mathrm{MeV}$ excitation energy has been tentatively associated to a transition from liquid to gas. A first order phase transition can be unambiguously defined in a finite system as an abnormal curvature of a thermodynamic potential e.g. a convexity of the entropy [2]. When this anomaly affects the energy axis, the heat capacity becomes negative. The first experimental signatures of negative heat capacities have been recently reported for atomic clusters [3, 4] and nuclei [5, [6]. The results on $\mathrm{Na}$ clusters have been related to the melting of $N a$-matter $[3$. For nuclei the link with bulk properties is problematic since the long range Coulomb interaction forbids the definition of a thermodynamic limit. Nuclear matter is a theoretical construction assuming the proton charge to be zero and one may worry about the connection between the properties of charged nuclei and the thermodynamics of uncharged nuclear matter. In this letter, we propose a systematic way to perform such a connection.

Let us develop the link between charged and uncharged systems. The long range Coulomb interaction can be written as $E_{c}=q^{2} V_{c}$ where $q$ represents the proton charge (in electron charge unit $q=1$ ) while $V_{c}=\sum_{p<p^{\prime}} \alpha /\left|\mathbf{r}_{p}-\mathbf{r}_{p^{\prime}}\right|$ with $\alpha=e^{2} / 4 \pi \epsilon_{0}$. The total energy of the system $E$ can thus be splitted into two terms $E_{t o t}=E_{n}+E_{c}=E_{n}+q^{2} V_{c}$. If the system is in contact with a heat bath (canonical ensemble) we can sort the events as a function of both $E_{n}$ and $V_{c}$ :

$$
p_{\beta}\left(E_{n}, V_{c}\right)=\frac{1}{Z_{\beta}} W\left(E_{n}, V_{c}\right) e^{-\beta E_{n}-\beta q^{2} V_{c}} .
$$

The canonical total energy distribution is an integration of the generalized distribution (1): $p_{\beta}\left(E_{t o t}\right)=$ $\int d V_{c} p_{\beta}\left(E_{t o t}-q^{2} V_{c}, V_{c}\right)$. The microcanonical entropy, $S_{t o t}\left(E_{t o t}\right)=\log W_{t o t}\left(E_{t o t}\right)$, is also directly related to $p_{\beta}\left(E_{t o t}\right): S_{t o t}\left(E_{t o t}\right)=\log p_{\beta}\left(E_{t o t}\right)+\beta E_{t o t}+\log Z_{\beta}$. Eq. (11) naturally leads to the definition of a generalized entropy $S\left(E_{n}, V_{c}\right)=\log W\left(E_{n}, V_{c}\right)$. The standard entropy can be obtained by a projection of the density of states on the total energy $E_{t o t}=E_{n}+q^{2} V_{c}$ axis

$$
W_{t o t}\left(E_{t o t}\right)=\int d V_{c} W\left(E_{t o t}-q^{2} V_{c}, V_{c}\right)
$$

One can also notice that the projection of $W\left(E_{n}, V_{c}\right)$ on the $E_{n}$ axis provides the entropy of the uncharged system

$$
S_{n}\left(E_{n}\right)=\log W_{n}\left(E_{n}\right)=\log \int d V_{c} W\left(E_{n}, V_{c}\right)
$$

Since we have introduced two observables $\left(E_{n}, V_{c}\right)$, it is natural to extend the canonical ensemble by introducing two Lagrange multipliers $\beta_{n}, \beta_{c}$ leading to the distribution of events in the two-dimensional observables space

$$
p_{\beta_{n} \beta_{c}}\left(E_{n}, V_{c}\right)=\frac{1}{Z_{\beta_{n} \beta_{c}}} W\left(E_{n}, V_{c}\right) e^{-\beta_{n} E_{n}-\beta_{c} q^{2} V_{c}}
$$

where $Z_{\beta_{n} \beta_{c}}$ is the partition sum of this multi-canonical ensemble corresponding to the independent observation of the two components $E_{n}, V_{c}$ of the energy. In the cases $\beta_{n}=\beta_{c}$ eq.(2) reduces to the usual canonical distribution of interacting charged particles (1) while $\beta_{c}=0$ corresponds to systems not affected by the Coulomb force. All intermediate values $0<\beta_{c}<\beta_{n}$ give rise to interpolating ensembles between the charged and the uncharged case, in the same way as the gaussian ensemble 7] gives a continuous interpolation between the microcanonical and the canonical ensemble. The multicanonical ensemble can also be considered as the canonical ensemble at temperature $\beta_{n}^{-1}$ of particles charged with an effective charge $\bar{q}^{2} / q^{2}=\beta_{c} / \beta_{n}$. In fact we can introduce an effective energy as $E_{\text {eff }}=E_{n}+\bar{q}^{2} V_{c}$. Then the distribution of the effective energy is a canonical distribution with a Boltzmann factor $e^{-\beta_{n} E_{\text {eff }}}$. Considering $\beta_{c}=0$ (or $\beta_{c} \rightarrow 0$ faster then the increase of the charge when the volume of the system is increased), the multicanonical ensemble is a way to study the thermodynamic limit.

Let us now focus on the question of (first order) phase transitions by studying the convex region of the entropy $S\left(E_{c}, E_{n}\right)$. Eq.(2) shows that the curvature matrix of the entropy and of the probability distribution 
$\log p_{\beta_{n} \beta_{c}}\left(E_{c}, V_{n}\right)$ are the same for every set of Lagrange multipliers $\beta_{n}, \beta_{c}$. This implies that the whole thermodynamics of the uncharged system can be (at least in principle) completely reconstructed from the only knowledge of the distribution probability of the charged system at an arbitrary temperature and pressure. For example, the uncharged system entropy can be deduced within a constant from the statistical distribution of the charged system by projecting out the Coulomb energy after a proper Boltzmann weighting

$$
S_{n}\left(E_{n}\right)=\log \left(\int d V_{c} e^{\beta_{c} q^{2} V_{c}} p_{\beta_{n} \beta_{c}}\left(E_{n}, V_{c}\right)\right)-\beta_{n} E_{n} .
$$

The only limitation can be a practical one for experiments or simulations: to accumulate enough events at every location.

In order to illustrate these general ideas and make some connections to the nuclear multifragmentation experiments, we have made some calculations in the multicanonical ensemble with a statistical multifragmentation model [8]. The model describes a multifragmentation event as an ensemble of spherical, non overlapping fragments interacting through their mutual Coulomb repulsion. The masses of the fragments are parametrized from a charged liquid drop model while their excitation energies are taken from a Fermi gas distribution with a high energy cut-off. The fragment translational degrees of freedom are treated classically. Each event is characterized by its total mass and charge number (i.e. the size of the disassembling nuclear source), its total energy that can be decomposed in a Coulomb $E_{c}$ part that includes also Coulomb effects on the binding energy, and a non Coulomb one that corresponds to the nuclear interaction inside fragments plus the translational energy. Each event can also be associated to a spatial extension, given by the box ('freeze-out' volume) that contains all the fragments. In an open system as a multifragmenting nucleus, this volume is not fixed but can fluctuate from one event to the other. If the average volume at freeze-out is finite, we can treat the volume as an extra observable known in average taking this constraint into account as an additional Lagrange multiplier $\lambda=\beta P$ where $\beta=\beta_{n}$ is the inverse temperature and $P$ has the dimension of a pressure [9, 10]. The multi-canonical probability (2) is then evaluated with a Metropolis technique [8].

The resulting distribution of events can then be plotted in the $\left(E_{n}, V_{c}\right)$ plane or equivalently in the $\left(E=E_{n}+\right.$ $\left.V_{c}, V_{c}\right)$ plane. When the Coulomb interaction is at play $(\bar{q}=1)$ the total energy is simply the $E$ axis. When the Coulomb energy is not effective $(\bar{q}=0)$ the relevant energy axis is the $E-V_{c}$ one i.e. the second bisector.

In the isobar ensemble we are considering, energy is expected to be an order parameter for the ordinary liquidgas phase transition (in the uncharged system), imply- ing that, for all pressures below the critical one and for all temperatures in the transition region, we expect the event cloud to separate into two components (phases) along the energy axis [2].

FIG. 1: Event distribution in the Coulomb energy versus total energy plane and relative projections with $\lambda=$ $3 \cdot 10^{-4} \mathrm{fm}^{-3}$ for a system of total mass number $A=50$ and atomic number $Z=23$ in the isobar multicanonical multifragmentation model. Levels of grey and full lines: $\beta_{n}^{-1}=3.7 \mathrm{MeV}, \beta_{c}=0$; contour lines and dashed lines: $\beta_{n}^{-1}=\beta_{c}^{-1}=3.1 \mathrm{MeV}$.

Figure 1 shows the distribution of events for a system of 50 particles at a subcritical pressure $\beta P=3 \cdot 10^{-4} \mathrm{fm}^{-3}$ in the Coulomb energy $V_{c}$ and total energy $E$ (calculated respect to the ground state of the source) plane. The charged case $\beta_{c}=\beta_{n}$ and the uncharged one $\beta_{c}=0$ are displayed together with their projections on the two axes.

Let us look at the uncharged case first. The topology of events is characteristic of a first order phase transition, with an accumulation of events at low excitation energy and high Coulomb energy (compact configurations or "liquid" phase) and an accumulation at high energy and low Coulomb (rarefied configurations or "gas" phase). The distance between the two peaks along the nuclear energy axis $E-V_{c}$, measures the latent heat of the transition while the region of inverted curvature is related to a negative heat capacity in the microcanonical ensemble. The representation in the $\left(E, V_{c}\right)$ plane reveals that the liquid peak is constituted by a series of structures. They can be attributed to different channel openings associated to similar excitation energies but corresponding to different proton partitions leading to different $V_{c}$. They constitute the microscopic origin of the global collective phase change that in the bulk limit will become the ordinary liquid-gas phase transition.

FIG. 2: Event distribution in the Coulomb energy versus total energy plane with $\lambda=5 \cdot 10^{-3} \mathrm{fm}^{-3}$ for a system of total mass number $A=50$ and atomic number $Z=23$ in the isobar multicanonical multifragmentation model. Levels of grey: $\beta_{n}^{-1}=7.5 \mathrm{MeV}, \beta_{c}=0$; contour lines in the left panel: $\beta_{n}^{-1}=\beta_{c}^{-1}=6.7 \mathrm{MeV}$; contour lines in the right panel: distribution with Coulomb reweighted to get the uncharged case.

The event distribution of the charged system in Figure 1 shows globally the same structure as in the uncharged case, with two important differences. First, to have the same height in the two relative maxima the temperature must be lower. The decrease of the transition temperature is due to the repulsive character of the Coulomb interaction [10]. Second, even if the phase transition is 
still clearly visible in the bidimensional representation, this is not true any more for the projection over the total energy axis $(E)$. Indeed, the fact that the distance between the peaks (latent heat) is comparable to the width of each peak implies that the bimodality is hardly perceptible in the energy direction. The usual interpretation is then the lowering of the critical point due to the Coulomb interaction. The complete bidimensional information of Figure 1 demonstrates that this conclusion is not completely correct. Indeed while the event distribution is almost unchanged, the projection axis is different in the charged case and the best order parameter (i.e. the direction that separates at best the two phases) is now almost perpendicular to the energy direction. Introducing a better observable playing the role of the order parameter, $O=a E+b V_{c}$, would thus restore the overall picture of the first order phase transition even in the charged system.

The introduction of the charge is just a reweighting in the occupation probabilities of the very same entropy surface. Indeed, if we consider a charged system at the temperature $\beta_{1}^{-1}$ described by the distribution $p_{\beta_{1}}^{c} \equiv p_{\beta_{n}=\beta_{1}, \beta_{c}=\beta_{1}}$, the distribution $p_{\beta_{2}}^{n} \equiv p_{\beta_{n}=\beta_{2}, \beta_{c}=0}$ at the temperature $\beta_{2}^{-1}$ in the case where the Coulomb interaction is not active is given (within a normalization) by

$$
p_{\beta_{2}}^{n}\left(E_{n}, V_{c}\right) \propto p_{\beta_{1}}^{c}\left(E_{n}, V_{c}\right) e^{\left(\beta_{1}-\beta_{2}\right) E_{n}+\beta_{1} q^{2} V_{c}}
$$

An example of the reconstruction of an uncharged distribution, starting from a calculation including the Coulomb interaction is shown in figure 2. The perfect reproduction of the distribution demonstrates that, even if the inclusion of Coulomb leads the system to occupy the phase space in a different way, the density of states $W$ itself does not change and the same information about its convexity anomalies and phase transitions properties can be inferred from the two calculations.

FIG. 3: Event distribution in the Coulomb energy versus total energy plane with $\lambda=1.26 \cdot 10^{-3} \mathrm{fm}^{-3}$ for a system of total mass number $A=200$ and atomic number $Z=82$ in the isobar multicanonical multifragmentation model. Levels of grey: $\beta_{n}^{-1}=5.6 \mathrm{MeV}, \beta_{c}=0$; contour lines (from top to bottom): $\beta_{n}^{-1}=\beta_{c}^{-1}=3 ., 3.7,4.4,4.8 \mathrm{MeV}$. Black contour lines: $\beta_{n}^{-1}=5 . \mathrm{MeV}$ and partially screened charge $\bar{q}=0.14$.

From a practical point of view, this reconstruction is possible only for the energy states where the population sample is statistically significant; if the probability distributions are concentrated in very different locations in the observables space the size of the sample needed will become increasingly (and soon prohibitively) high. This more complicated situation happens when the average partitions are very different in the charged and uncharged case. This is indeed the case if we consider heavily charged systems, for which the Coulomb distortions get more appreciable. Figure 3 shows the event distribution for a heavy system composed of $A=200$ particles. When the Coulomb interaction is neglected the situation gets closer to the expected behavior in the bulk: the channel openings are not recognizeable any more and only the collective liquid-like to gas-like state change survives, with a minimum between the two phases getting deeper. If Coulomb is switched on, the event topology drastically changes. A much smaller portion of phase space is populated by any calculation at a single temperature, and this stays true if the pressure is changed. The event distribution turns around the first order phase transition without diving into the coexistence region. The external edge of the anomalous curvature region is only touched by the calculations at the lowest temperature, pointing to an order parameter almost perfectly perpendicular to the energy direction. This residual bimodality corresponds to the opening of asymmetric fission, and is the only rest of the liquid-gas phase transition in heavily charged systems. Since the average transformations in the $\left(E, V_{c}\right)$ plane are so far away, the reconstruction of the neutral matter phase diagram requires a prohibitively high statistics and cannot be performed for too heavy systems.

In conclusion, in this letter we have introduced a multicanonical formalism that allows a direct mapping between a system composed of particles with or without an electric charge. This general framework allows to study the effect of a long range non saturating force as the Coulomb interaction on a first order phase transition. Such an interaction is seen to deform the distribution of events in the space of observables but its strongest effect is to rotate the energy axis away from the order parameter. This effect is responsible for the apparent reduction or suppression of the coexistence region. If the system is not too heavily charged, the nuclear energy remains well correlated with the order parameter and the transition phenomenology is not strongly affected by the presence of the Coulomb field. The Coulomb simply manifests itself as a lowering of the critical parameters which is normal since the binding energy is reduced. The convexity anomalies of the entropy can be traced back from the event distribution independently of the Coulomb interaction and the equations of state of the uncharged system can be obtained from a charged population sample if the Coulomb energy is measured on an event-by-event basis and the relevant phase space is sampled with enough statistics.

For heavily charged systems the considered statistical model predicts that, at low temperature, the events strongly differ from the uncharged partitions. Therefore the two thermodynamics can only be related at high energy when the gas phase is reached. Moreover in the charged system the transition from the liquid to gas goes around the coexistence region and so corresponds 
to a cross over and not to a first order phase transition. However, the size at which this phenomenon appears is model dependent and more investigations are needed to get quantitative predictions.

Finally we would like to stress that all the interpolating ensembles with $0<\beta_{c}<\beta_{n}$ (see black contour lines in figure 3) may also be interesting to model experimental situations in which the different parts of the energy are not equilibrated. This may happen in actual reactions since the relaxation time depends critically on the range of the force [1]. For example, many scenarii of multifragmentation involve spinodal instabilities which are weakly sensitive to the Coulomb force 12]. Being very chaotic, spinodal decomposition may then well populate a complete phase space for which $E_{n}$ is the important physical quantity and not $E_{t o t}$. This would correspond to a $\beta_{c} \approx 0$ case. Such a situation might be suggested by the insensitivity to the total system charge of the fragmentation pattern observed in multifragmentation data from central reactions and predicted in spinodal decomposition simulations [13]. However, this question requires a strong theoretical and experimental effort which goes beyond the scope of the present article.

[1] P.J. Siemens, Nature 305 (1983) 410; A. D. Panagiotou et al., Phys. Rev. Lett. 52, 496 (1984).
[2] Ph.Chomaz, F.Gulminelli, V.Duflot, Phys.Rev.E 64 (2001) 046114; T.Dauxois et al., 'Dynamics and Thermodynamics of systems with long range interactions', Lecture Notes in Physics vol.602, Springer (2002).

[3] M. Schmidt et al., Phys.Rev.Lett.86(2001)1191.

[4] F.Gobet et al., Phys. Rev. Lett.89 (2002)183403.

[5] S.Siem et al., Phys. Rev. C65 (2002) 044318.

[6] M. D'Agostino et al., Phys. Lett.B 473 (2000) 219; ibid, Nucl. Phys. A 699 (2002) 795.

[7] M.S.S.Challa and J.H.Hetherington, Phys.Rev.Lett. 60 (1988) 77.

[8] Al. H. Raduta and Ad. R. Raduta, Phys. Rev. C 55, 1344 (1997); ibid 56, 2059 (1997); ibid 61, 034611 (2000).

[9] F. Gulminelli, Ph. Chomaz and V.Duflot, Europhys. Lett. 50 (2000) 434.

[10] Al. H. Raduta, Ad. R. Raduta, Nucl. Phys. A703 (2002) 876.

[11] V. Latora, A. Rapisarda, C. Tsallis, Phys. Rev. E64 (2001) 056134 .

[12] M.Colonna et al., Phys. Rev. Lett. 88 (2002) 122701; B.Borderie et al., Phys. Rev. Lett. 86 (2001) 3252.

[13] M. F. Rivet et al., Phys. Lett. B430 (1998) 217; J. D. Frankland et al., Nucl.Phys. A689 (2001) 905. 
This figure "fig1.png" is available in "png" format from: http://arxiv.org/ps/nucl-th/0306064v1 
This figure "fig2.png" is available in "png" format from: http://arxiv.org/ps/nucl-th/0306064v1 
This figure "fig3.png" is available in "png" format from: http://arxiv.org/ps/nucl-th/0306064v1 